\title{
Direct Frequency Comb Laser Cooling and Trapping
}

\author{
A. M. Jayich, ${ }^{*}$ X. Long, and W. C. Campbell \\ Department of Physics and Astronomy, University of California, Los Angeles, Los Angeles, \\ California 90095, USA \\ and California Institute for Quantum Emulation, Santa Barbara, California 93106, USA
}

(Received 11 May 2016; published 10 October 2016)

\begin{abstract}
Ultracold atoms, produced by laser cooling and trapping, have led to recent advances in quantum information, quantum chemistry, and quantum sensors. A lack of ultraviolet narrow-band lasers precludes laser cooling of prevalent atoms such as hydrogen, carbon, oxygen, and nitrogen. Broadband pulsed lasers can produce high power in the ultraviolet, and we demonstrate that the entire spectrum of an optical frequency comb can cool atoms when used to drive a narrow two-photon transition. This multiphoton optical force is also used to make a magneto-optical trap. These techniques may provide a route to ultracold samples of nature's most abundant building blocks for studies of pure-state chemistry and precision measurement.
\end{abstract}

DOI: 10.1103/PhysRevX.6.041004

Subject Areas: Atomic and Molecular Physics

\section{INTRODUCTION}

High-precision physical measurements are often undertaken close to absolute zero temperature to minimize thermal fluctuations. For example, the measurable properties of a room temperature chemical reaction (rate, product branching, etc.) include a thermally induced average over a large number of reactant and product quantum states, which masks the unique details of specific reactant-product pairs. Doppler laser cooling with continuous wave lasers is a robust method to reduce the random motion of atoms [1] and molecules [2]. With some added complexity, the same laser light can be made to spatially confine the atoms in a magneto-optical trap (MOT) [3,4]. The resulting sub-kelvin gas-phase atoms can then be studied and controlled with high precision, which has led to recent advances in quantum information $[5,6]$, the search to understand dark energy [7], and quantum sensors [8]. Laser cooling and trapping has recently enabled the measurement and control of ultracold chemical reactions at a new level of detail $[9,10]$ with molecules made from alkali atoms that are well suited to cw laser technology.

While the prospects of comprehensive precision spectroscopy and pure state resolution of arbitrary chemical reactions is enticing, Doppler cooling is limited by the availability of $\mathrm{cw}$ lasers to a subset of atoms and molecules that have convenient internal structure. In particular, the lack of sufficiently powerful cw lasers in the deep

*jayich@gmail.com

Published by the American Physical Society under the terms of the Creative Commons Attribution 3.0 License. Further distribution of this work must maintain attribution to the author(s) and the published article's title, journal citation, and DOI. ultraviolet means that laser cooling and trapping is not currently available for the most prevalent atoms in organic chemistry: hydrogen, carbon, oxygen, and nitrogen. Because of their simplicity and abundance, these species, with the addition of antihydrogen, likewise play prominent roles in other scientific fields such as astrophysics [11] and precision measurement [12], where the production of cold samples could help answer fundamental outstanding questions [13-15].

In contrast to cw lasers, mode-locked (ML) lasers have very high instantaneous intensity and can, therefore, be efficiently frequency summed to the UV. However, the spectrum of a ML laser consists of many evenly spaced spectral lines (an optical frequency comb) spanning a bandwidth much larger than a typical Doppler shift, and ML lasers have, therefore, found very little use as control tools for cooling the motion of atoms and molecules [16]. Doppler cooling with combs has been investigated in a mode where each atom interacts with only one or two comb teeth at a time, which uses only a small fraction of the laser's total power [17-21]. Here, following the observation of a pushing force by Marian et al. [22] and a proposal by Kielpinski [23], we utilize a coherent effect in far-detuned ML two-photon transitions [24] to laser cool atoms with all of the comb teeth contributing in parallel to enhance the scattering rate (Fig. 1). This technique is designed to utilize the high UV conversion efficiency of ML lasers without wasting any of the resulting UV power, and opens the door to laser cool $\mathrm{H}, \mathrm{C}, \mathrm{N}, \mathrm{O}$, and antihydrogen $(\overline{\mathrm{H}})$, species for which single-photon laser cooling is beyond the reach of even ML lasers [23]. We extend these ideas to create a magneto-optical trap, and find that the density of the comb spectrum introduces no measurable effects in our system, demonstrating that it may be possible to create MOTs of these species using this technique. 

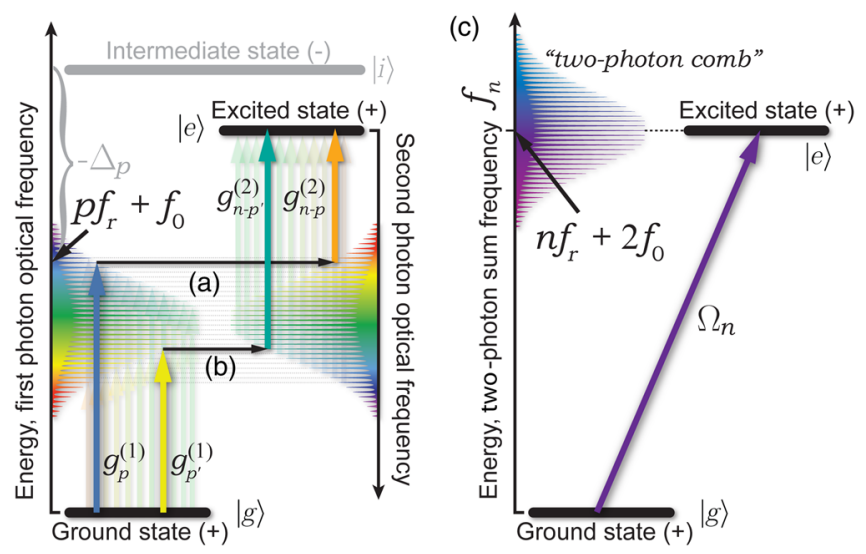

FIG. 1. Constructive interference of multiple paths in a twophoton transition driven by a transform-limited optical frequency comb. All pairs of comb teeth whose sum frequency matches the excited state energy interfere constructively to excite atoms. Two example pairs are shown as (a) and (b), and the effective two-level system that results from the sum is shown in (c). Every tooth of the resulting "two-photon comb" of resonant coupling strength $\Omega$ leverages the full power of all of the optical frequency comb teeth through this massively-parallel constructive interference.

\section{THEORY}

A simple model can be used to describe the interaction between three-level atoms and an optical frequency comb for two-photon laser cooling and trapping (see Fig. 1 and Ref. [25] for details). The two-photon coupling strength between the ground and excited states in this case will also be a comb [the "two-photon comb" shown in Fig. 1(c)], the $n$th tooth of which is associated with a frequency $f_{n}=n f_{r}+2 f_{0}$, where $f_{0}$ is the carrier-envelope offset frequency of the optical comb and $f_{r} \equiv 1 / T_{r}$ is the pulse repetition rate. For a transform-limited ML laser, we can model the effective (time-averaged) resonant Rabi frequency of the $n$th tooth of this two-photon comb as

$$
\Omega_{n}=\sum_{p} \frac{g_{p}^{(1)} g_{n-p}^{(2)}}{2 \Delta_{p}},
$$

where $g_{p}^{(1)}$ is the resonant single-photon Rabi frequency for excitation from the ground $|g\rangle$ to the intermediate state $|i\rangle$ due to the $p$ th optical comb tooth and $g_{p}^{(2)}$ is the same quantity for excitation from the intermediate state $|i\rangle$ to the excited state $|e\rangle$ [Figs. 1(a) and 1(b)]. For chirped (as opposed to transform-limited) pulses, $g_{p}$ is complex and the phase of the product $g_{p}^{(1)} g_{n-p}^{(2)}$ becomes a function of $p$, which can result in destructive interference between multiple paths, reducing the magnitude of $\Omega_{n}$. The single-photon detuning from the intermediate state is $\Delta_{p}=p f_{r}+f_{0}-f_{\mathrm{gi}}$, where $f_{\mathrm{gi}}$ is the intermediate state's energy divided by Planck's constant $h$ (we take the energy of the ground state to be zero). If we denote by $N$ the index of the two-photon comb tooth closest to resonance (associated with the optical sum frequency $f_{N}=N f_{r}+2 f_{0}$ ) and the pulse duration is short compared to the excited state's lifetime $(\tau \equiv 1 / \gamma)$, we can approximate the resonant Rabi frequency of each two-photon comb tooth in the vicinity of resonance as being given by $\Omega_{N}$. In the limit of weak single-pulse excitation $\left(\Omega_{N} T_{r} \ll \pi\right)$, the timeaveraged excitation rate for an atom moving with velocity $v$ is given by (see Refs. $[19,20,25]$ )

$$
\gamma_{\mathrm{comb}}=\frac{\Omega_{N}^{2} T_{r}}{4} \frac{\sinh \left(\gamma T_{r} / 2\right)}{\cosh \left(\gamma T_{r} / 2\right)-\cos \left(\delta_{N}(v) T_{r}\right)},
$$

where $\delta_{N}(\boldsymbol{v}) \equiv 2 \pi\left(f_{N}-f_{\mathrm{ge}}-f_{N} \hat{\boldsymbol{k}} \cdot \boldsymbol{v} / c\right)$ is the detuning of the $N$ th two-photon comb tooth from two-photon resonance, $\hat{\boldsymbol{k}}$ is a unit vector pointing in the direction of laser propagation, and $f_{\mathrm{ge}}$ is the energy of the excited state divided by $h$. If both the detuning $\delta_{N}(\boldsymbol{v})$ and natural linewidth $\gamma$ are small compared to the comb tooth spacing $\left(2 \pi f_{r}\right)$, this two-photon comb can be treated as having only a single tooth (monochromatic interaction) with a two-photon Rabi frequency of $\Omega_{N}$. Most of the work we describe here takes place once the atoms are fairly cold $\left(k v \ll 2 \pi f_{r}\right)$ in this "single two-photon tooth limit," which gives rise to an excitation rate of

$$
\gamma_{N}=\frac{\Omega_{N}^{2}}{\gamma} \frac{1}{1+\left[2 \delta_{N}(\boldsymbol{v}) / \gamma\right]^{2}} .
$$

Since the ac Stark shifts from the proximity of the intermediate state to the optical photon energy are the same order of magnitude as $\Omega_{N}$, they can be neglected compared to the linewidth in the low-saturation limit. For cases where a single laser photon has enough energy to photoionize an excited atom, since both the time-averaged excitation rate and the time-averaged photoionization rate from the excited state depend only upon the time-averaged intensity, the average ionization rate is exactly the same as for a $\mathrm{cw}$ laser with the same average frequency and timeaveraged power [23].

Using this simplification, an algebraic model for Doppler cooling can be constructed for the degenerate two-photon case (as opposed to two-color excitation [26]) to estimate the Doppler temperature. We assume that the laser's center frequency is near $f_{\mathrm{ge}} / 2$ and that the single tooth of interest in the two-photon comb can be characterized by a twophoton saturation parameter $s_{N} \equiv 2 \Omega_{N}^{2} / \gamma^{2} \ll 1$. For slow atoms $(k v \ll \gamma)$, the cooling power of a 1D, two-photon optical molasses detuned $\gamma / 2$ to the red side of two-photon resonance is given by the same expression as the singlephoton cw laser cooling case, $\partial E /\left.\partial t\right|_{\text {cool }}=-s_{N} \hbar \omega_{\mathrm{ge}}^{2} v^{2} / c^{2}$, where $\omega_{\text {ge }} \equiv 2 \pi f_{\text {ge }}$. The heating caused by momentum kicks from absorption is likewise identical to the $\mathrm{cw}$ singlephoton expression, $\partial E /\left.\partial t\right|_{\text {heat }, \text { abs }}=s_{N} \gamma \hbar^{2} \omega_{\mathrm{ge}}^{2} / 4 m c^{2}$. 
The heating caused by spontaneous emission, however, is modified by both the multiphoton nature of the emission and the details of excitation by a comb as follows. First, the decay of the excited state is likely to take place in multiple steps due to the parity selection rule, splitting the deexcitation into smaller momentum kicks that are unlikely to occur in the same direction, reducing the heating. Second, two-photon laser cooling with counterpropagating $\mathrm{cw}$ laser beams adds heating in the form of Doppler-free (two-beam) excitations [27], which produce no cooling force in 1D but do cause heating through the subsequent spontaneous emission. By using a comb, however, one can easily eliminate these Doppler-free transitions through timing by ensuring that pulses propagating in different directions do not hit the atoms simultaneously. In the frequency domain, this delay produces a frequency-dependent phase shift of the frequency comb for the second photon [shown on the right-hand side of Figs. 1(a) and 1(b)], destroying the coherent addition of comb teeth pairs necessary to drive the transition. The net result is that the heating rate from spontaneous emission for two-photon laser cooling with an optical frequency comb can be modeled by

$$
\left.\frac{\partial E}{\partial t}\right|_{\text {heat,spon }}=s_{N} \gamma \frac{\hbar^{2} \omega_{\mathrm{ge}}^{2}}{8 m c^{2}} .
$$

The balance between the cooling power and the sum of these heating powers occurs at the Doppler temperature for two-photon laser cooling with an optical frequency comb:

$$
T_{D}=\frac{3}{4} \frac{\hbar \gamma}{2 k_{B}},
$$

where $k_{B}$ is the Boltzmann constant.

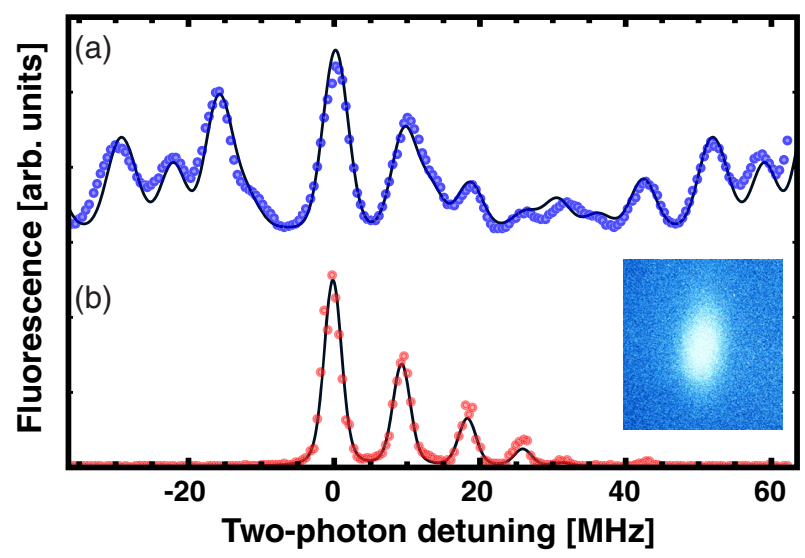

\section{EXPERIMENTAL RESULTS}

As a first experimental test of direct frequency comb two-photon cooling and trapping, we report a demonstration of the technique using rubidium atoms. For the $5^{2} S_{1 / 2} \rightarrow 5^{2} D_{5 / 2}$ transition in rubidium [Fig. 2(c)], the natural decay rate of the excited state is $\gamma / 2 \pi=667 \mathrm{kHz}$ [28]. Equation (5) gives a Doppler cooling limit of $12 \mu \mathrm{K}$, which is also true in 3D for a ML laser with noncolliding pulses. In this work, we apply cooling in 1D with spontaneous emission into 3D, and our effective transition linewidth must also be taken into account (see Ref. [25]), which yields a predicted Doppler limit of $31 \mu \mathrm{K}$ for this system, considerably colder than the single-photon $5^{2} S_{1 / 2} \rightarrow 5^{2} P_{3 / 2}$ 3D Doppler limit of $146 \mu \mathrm{K}$.

The optical frequency comb in this work is generated from a Ti:sapphire laser emitting 2-5-ps pulses (less than $500 \mathrm{GHz}$ bandwidth) at $778 \mathrm{~nm}$ at a repetition rate of $f_{r}=81.14 \mathrm{MHz}$.

We prepare an initial sample of $\approx 10^{785} \mathrm{Rb}$ atoms using a standard cw laser MOT at $780 \mathrm{~nm}$. The magnetic field and the $\mathrm{cw}$ laser cooling light are then turned off, leaving the atoms at a temperature typically near $110 \mu \mathrm{K}$. A weak cw "repump" laser is left on continuously to optically pump atoms out of the $F_{g}=2$ ground state, and has no measurable direct mechanical effect. Each ML beam typically has a time-averaged power of $(500 \pm 50) \mathrm{mW}$ and a diameter of $(1.1 \pm 0.1) \mathrm{mm}$. After illumination by the ML laser, the atoms are allowed to freely expand and are subsequently imaged using resonant $\mathrm{cw}$ absorption to determine their position and velocity distributions.

By monitoring the momentum transfer from a single ML beam [Figs. 2(e) and 2(f)], we measure a resonant excitation rate of $\gamma_{\text {scatt }}=(6500 \pm 700) \mathrm{s}^{-1}$. Our transformlimited theoretical estimate from Eq. (1) and our laser
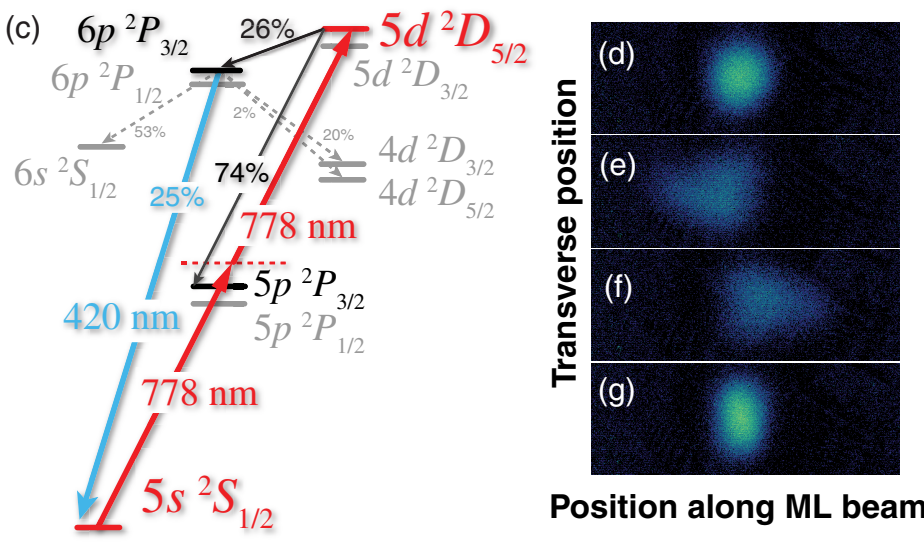

FIG. 2. Laser-induced fluorescence spectrum of the $5 S \rightarrow 5 D$ two-photon transition driven by an optical frequency comb. (a) Spectrum from a natural abundance vapor cell and (b) a ${ }^{85} \mathrm{Rb} \mathrm{cw}$ MOT and collected 420-nm light (inset). Solid curves are theory fitted for (a) Gaussian and (b) Voigt line shapes. These spectra repeat with a period of $f_{r}=81.14 \mathrm{MHz}$ on the horizontal axis. (c) Relevant levels of rubidium. (d)-(g) Absorption images of the atom cloud after free expansion following (d) no ML illumination, (e) ML illumination from the right, (f) left, and (g) both directions, detuned to the red of resonance. Mechanical forces are evident in (e) and (f), and the narrowing of the velocity distribution in the horizontal direction in ( $\mathrm{g}$ ) is the hallmark of cooling. 
parameters gives $(13000 \pm 2000) \mathrm{s}^{-1}$. Since the twophoton Rabi frequency is inversely proportional to the pulse time-bandwidth product [29], this suggests that there is residual chirp that is increasing the time-bandwidth product by a factor of $\approx \sqrt{2}$. The measured rate is well above the threshold needed to support these atoms against gravity $\left(\approx 810 \mathrm{~s}^{-1}\right)$, which suggests that $3 \mathrm{D}$ trapping should be possible with additional laser power for the inclusion of four more beams.

We observe Doppler cooling and its dependence on twophoton detuning by applying counterpropagating linearly polarized ML beams to the atom cloud for $4 \mathrm{~ms}$ in zero magnetic field. By fitting the spatial distribution of the atoms (see Ref. [25]), we extract a 1D temperature, shown in Fig. 3. The solid curve is based on the algebraic model used above to derive the Doppler limit and is fit for a resonant single-beam excitation rate of $(4800 \pm 400) \mathrm{s}^{-1}$ and linewidth $\gamma_{\text {eff }} / 2 \pi=(1.88 \pm 0.07) \mathrm{MHz}$ [see Figs. 2(a) and 2(b) and Ref. [25] for a discussion of finite linewidth], consistent with the single-beam recoil measurements and laser power fluctuations discussed above. We realize a minimum temperature of $(57 \pm 2) \mu \mathrm{K}$ (Fig. 3, inset). However, the reduced temperature is hotter than the

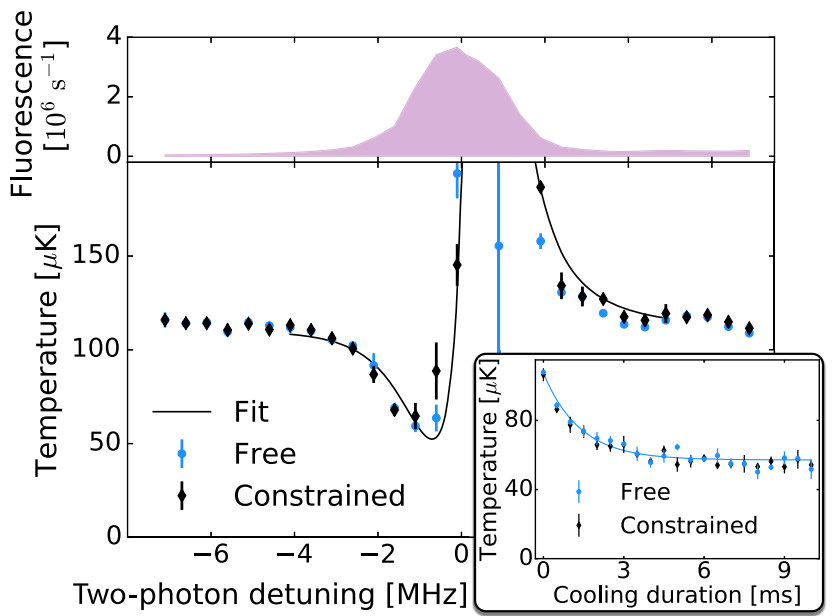

FIG. 3. Detuning dependence of 420-nm fluorescence (top) and the resulting temperature (bottom) of rubidium atoms laser cooled by an optical frequency comb on a two-photon transition. The solid curve is fit for scattering rate, effective linewidth, and detuning offset of data analyzed with the aid of a Monte Carlo technique (data labeled "Constrained"; see Ref. [25]). The same data are also analyzed using a free-expansion model ("Free"), and agree well with the Monte Carlo assisted analysis. Inset: Temperature versus time when the laser detuning is optimized for cooling, giving a minimum temperature of $(57 \pm 2) \mu \mathrm{K}$. The time decay of the 420-nm fluorescence shows that atoms leave the interaction region due to transverse motion in $(4.0 \pm 0.3) \mathrm{ms}$, but steady-state $1 \mathrm{D}$ temperature is reached in $\tau=(1.28 \pm 0.09) \mathrm{ms}$. Error bars are statistical over repeated measurements, and do not include the systematic effect of beam mode mismatch, discussed in text. expected Doppler limit of $31 \mu \mathrm{K}$ for our system (see Ref. [25]). We find experimentally that the temperature inferred from free-expansion imaging is highly sensitive to beam alignment, and therefore suspect the discrepancy is due to imperfect balancing of the forward and backward scattering forces at some locations in the sample [30]. We calculate that the scattering rate due to single-photon excitation on $5 S \rightarrow 5 P$ under these far-detuned conditions is of order $\approx 1 \mathrm{~s}^{-1}$, which would contribute a negligible amount of force in $4 \mathrm{~ms}$. The absence of observable singlephoton processes from possible near-resonant optical comb teeth is further evident in the period of the frequency dependence of Figs. 2(a), 2(b), and 3, which repeat every $f_{r}$ (as opposed to $2 f_{r}$, which would be expected for resonant single-photon processes [22]).

To investigate the feasibility of using this technique to make a MOT, a quadrupole magnetic field with a gradient of $7.7 \mathrm{G} / \mathrm{cm}$ is introduced and the ML beam polarizations are set to drive $\sigma^{ \pm}$transitions in the standard single-photon cw MOT configuration [3] to make a 1D MOT. We displace the atom cloud from the trap center and monitor the atoms as they are pushed toward the trap center, as shown in Fig. 4. The system is modeled as a damped harmonic oscillator, and fitting the motion of the atoms yields a trapping frequency of $\nu_{\mathrm{MOT}}=(40 \pm 9) \mathrm{Hz}$ and a cyclic damping rate of $(37 \pm 4) \mathrm{Hz}$. These MOT parameters imply a resonant excitation rate of $(7000 \pm 1000) \mathrm{s}^{-1}$ and an effective magnetic line shift of $(0.5 \pm 0.2) \mu_{B}$. The average of the calculated line shifts for all $\Delta m_{F}=+2\left(\sigma^{+} \sigma^{+}\right)$transitions would be $1.2 \mu_{B}$ for $F_{g}=3 \rightarrow F_{e}=5$. By measuring the polarization of the beam before and after the vacuum chamber for each pass, we infer that the fraction of the laser power with the nominally desired polarization at the location of the atoms is $97 \%$ for the forward beam and $87 \%$ for the retroreflected

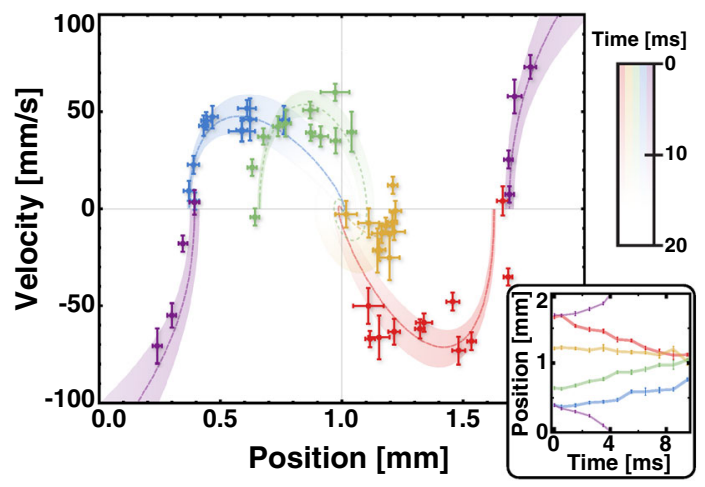

FIG. 4. Phase space (and position space, inset) trajectories for atoms trapped in a two-photon, optical frequency comb MOT. Smooth curves are fits to a damped harmonic oscillator, with fit uncertainties shown as bands. Purple features show the behavior when the ML beam polarizations are intentionally reversed and exhibit an anticonfining force. Error bars are statistical over repeated measurements. 
beam. In a simple 1D model with magnetic field (quantization axis) parallel to the light's $\hat{\boldsymbol{k}}$ vector, $\sigma^{+} \sigma^{+}, \sigma^{-} \sigma^{-}$, $\sigma^{+} \sigma^{-}$, and $\sigma^{-} \sigma^{+}$transitions can be driven. Including the average line shifts from all of the $\Delta m=0$ (from $\sigma^{+} \sigma^{-}$and $\sigma^{-} \sigma^{+}$transitions) and $\Delta m=-2$ transitions (from $\sigma^{-} \sigma^{-}$) weighted by our polarization measurements yields an effective magnetic line shift of $0.68 \mu_{B}$. During the $\approx 4 \mathrm{~ms}$ of ML illumination before the atoms' transverse motion causes them to exit the interaction volume, we do not detect any atom loss due to photoionization, consistent with the measured photoionization cross section [31].

\section{OUTLOOK}

Cooling and trapping of rubidium on a two-photon transition with a ML laser demonstrates that it may be possible to apply these techniques in the deep UV to laser cool and magneto-optically trap species such as $\mathrm{H}, \mathrm{C}, \mathrm{N}, \mathrm{O}$, and $\overline{\mathrm{H}}$. Because of low anticipated scattering rates, these species will likely need to be slowed using other means $[32,33]$. Direct comb laser cooling and trapping would then be used to cool them to the Doppler limit in a MOT.

For $\mathrm{H}$ and $\overline{\mathrm{H}}$, to minimize photoionization losses (of particular importance for $\overline{\mathrm{H}}$, see Ref. [25]), we propose two-photon cooling on $1 S \rightarrow 3 D$ at $205.0 \mathrm{~nm}$. By choosing a comb tooth spacing of $f_{r}=83.5 \mathrm{MHz}$, all six of the allowed hyperfine and fine structure transitions on $1 S \rightarrow 3 D$ can be driven simultaneously with a red detuning between $\gamma / 3$ and $\gamma$. We estimate a resonant excitation rate on $1 S \rightarrow 3 D$ of $\gamma_{\text {scatt }} \approx 1250 \mathrm{~s}^{-1}$ is achievable with demonstrated technology (see Ref. [25]). This excitation rate would produce an acceleration more than 50 times greater than that used in this work to make a MOT of rubidium.

Atomic oxygen has fine structure in its ${ }^{3} P$ ground state that spans a range of about $7 \mathrm{THz}$, so the comb's ability to drive multiple transitions at once is a crucial advantage. For the $\left(2 p^{4}\right)^{3} P \rightarrow(3 p)^{3} P$ transitions, a frequency comb centered at $226 \mathrm{~nm}$ with a 2-nm bandwidth would be able to drive simultaneous two-photon transitions for each fine structure component for a repetition rate near $79.79 \mathrm{MHz}$. Nitrogen cooling and trapping would proceed on the $\left(2 p^{3}\right)^{4} S_{3 / 2}^{o} \rightarrow(3 p)^{4} S_{3 / 2}^{o}$ two-photon transition at $207 \mathrm{~nm}$. Branching to the doublet manifold limits the total number of quartet excitations per atom to $\approx 10^{3}$, sufficient for laser cooling a hot, trapped sample [33] to the Doppler limit. The hyperfine structure in the ground state of ${ }^{15} \mathrm{~N}$ is split by $29 \mathrm{MHz}$, so excitation from a single two-photon tooth may be enough to produce both cooling and (off-resonant) hyperfine repumping. Carbon would likely require multiple combs [23], but each would operate on the same principles we investigate here.

This demonstration with rubidium confirms the essential aspects of laser cooling and trapping with frequency combs on two-photon transitions. Future work in extending this technique into the deep UV should be possible with the addition of frequency conversion stages for the ML light. In particular, as higher power UV frequency combs become available [34,35], the technology for laser cooling and trapping will extend the reach of these techniques to species that cannot currently be produced in ultracold form.

\section{ACKNOWLEDGMENTS}

The authors acknowledge discussions with Chris Monroe and Thomas Udem, and thank Jun Ye for encouraging us to pursue this work. The authors thank Anthony Ransford and Anna Wang for technical assistance, and Andrei Derevianko, Luis Orozco, and Trey Porto for comments on the manuscript. Initial work was supported by the U.S. Air Force Office of Scientific Research Young Investigator Program under Award No. FA9550-13-1-0167, with continuation supported by the NSF CAREER Program under Award No. 1455357. W. C. C. acknowledges support from the University of California Office of the President's Research Catalyst Award No. CA-15327861 .

[1] D. J. Wineland, R. E. Drullinger, and F. L. Walls, RadiationPressure Cooling of Bound Resonant Absorbers, Phys. Rev. Lett. 40, 1639 (1978).

[2] E. S. Shuman, J. F. Barry, and D. DeMille, Laser Cooling of a Diatomic Molecule, Nature (London) 467, 820 (2010).

[3] E. L. Raab, M. Prentiss, A. Cable, S. Chu, and D. E. Pritchard, Trapping of Neutral Sodium Atoms with Radiation Pressure, Phys. Rev. Lett. 59, 2631 (1987).

[4] M. T. Hummon, M. Yeo, B. K. Stuhl, A. L. Collopy, Y. Xia, and J. Ye, 2D Magneto-Optical Trapping of Diatomic Molecules, Phys. Rev. Lett. 110, 143001 (2013).

[5] J. J. García-Ripoll, P. Zoller, and J. I. Cirac, Quantum Information Processing with Cold Atoms and Trapped Ions, J. Phys. B 38, S567 (2005).

[6] I. Bloch, J. Dalibard, and S. Nascimbène, Quantum Simulations with Ultracold Quantum Gases, Nat. Phys. 8, 267 (2012).

[7] P. Hamilton, M. Jaffe, P. Haslinger, Q. Simmons, H. Müller, and J. Khoury, Atom-Interferometry Constraints on Dark Energy, Science 349, 849 (2015).

[8] S. M. Dickerson, J. M. Hogan, A. Sugarbaker, D. M. S. Johnson, and M. A. Kasevich, Multiaxis Inertial Sensing with Long-Time Point Source Atom Interferometry, Phys. Rev. Lett. 111, 083001 (2013).

[9] B. Zhu, B. Gadway, M. Foss-Feig, J. Schachenmayer, M. L. Wall, K. R. A. Hazzard, B. Yan, S. A. Moses, J. P. Covey, D. S. Jin, J. Ye, M. Holland, and A. M. Rey, Suppressing the Loss of Ultracold Molecules via the Continuous Quantum Zeno Effect, Phys. Rev. Lett. 112, 070404 (2014).

[10] M. H. G. de Miranda, A. Chotia, B. Neyenhuis, D. Wang, G. Quéméner, S. Ospelkaus, J. L. Bohn, J. Ye, and D. S. Jin, Controlling the Quantum Stereodynamics of Ultracold Bimolecular Reactions, Nat. Phys. 7, 502 (2011).

[11] E. Herbst, Chemistry in the Interstellar Medium, Annu. Rev. Phys. Chem. 46, 27 (1995). 
[12] R. Bluhm, V. A. Kostelecký, and N. Russell, CPT and Lorentz. Tests in Hydrogen and Antihydrogen, Phys. Rev. Lett. 82, 2254 (1999).

[13] J. Dutta, B. B. Nath, P. C. Clark, and R. S. Klessen, The Role of Three-Body $\mathrm{H}_{2}$ Formation in the Fragmentation of Primordial Gas, Mon. Not. R. Astron. Soc. 450, 202 (2015).

[14] P. H. Donnan, M. C. Fujiwara, and F. Robicheaux, A Proposal for Laser Cooling Antihydrogen Atoms, J. Phys. B 46, 025302 (2013).

[15] P. Hamilton, A. Zhmoginov, F. Robicheaux, J. Fajans, J. S. Wurtele, and H. Müller, Antimatter Interferometry for Gravity Measurements, Phys. Rev. Lett. 112, 121102 (2014).

[16] B. B. Blinov, R. N. Kohn, Jr., M. J. Madsen, P. Maunz, D. L. Moehring, and C. Monroe, Broadband Laser Cooling of Trapped Atoms with Ultrafast Pulses, J. Opt. Soc. Am. B 23, 1170 (2006).

[17] P. Strohmeier, T. Kersebom, E. Krüger, H. Nölle, B. Steuter, J. Schmand, and J. Andrä, Na-Atom Beam Deceleration by a Mode-Locked Laser, Opt. Commun. 73, 451 (1989).

[18] M. Watanabe, R. Ohmukai, U. Tanaka, K. Hayasaka, H. Imajo, and S. Urabe, Velocity Control of an $\mathrm{Yb}$ Beam by a Frequency-Doubled Mode-Locked Laser, J. Opt. Soc. Am. B 13, 2377 (1996).

[19] E. Ilinova, M. Ahmad, and A. Derevianko, Doppler Cooling with Coherent Trains of Laser Pulses and a Tunable Velocity Comb, Phys. Rev. A 84, 033421 (2011).

[20] D. Aumiler and T. Ban, Simultaneous Laser Cooling of Multiple Atomic Species Using an Optical Frequency Comb, Phys. Rev. A 85, 063412 (2012).

[21] J. Davila-Rodriguez, A. Ozawa, T. W. Hänsch, and T. Udem, Doppler Cooling Trapped Ions with a UV Frequency Comb, Phys. Rev. Lett. 116, 043002 (2016).

[22] A. Marian, M. C. Stowe, J. R. Lawall, D. Felinto, and J. Ye, United Time-Frequency Spectroscopy for Dynamics and Global Structure, Science 306, 2063 (2004).

[23] D. Kielpinski, Laser Cooling of Atoms and Molecules with Ultrafast Pulses, Phys. Rev. A 73, 063407 (2006).
[24] Y. V. Baklanov and V. P. Chebotayev, Narrow Resonances of Two-Photon Absorption of Super-Narrow Pulses in a Gas, Appl. Phys. 12, 97 (1977).

[25] See Supplemental Material at http://link.aps.org/ supplemental/10.1103/PhysRevX.6.041004 for details on two-photon cooling and its application to hydrogen.

[26] S. Wu, T. Plisson, R. C. Brown, W. D. Phillips, and J. V. Porto, Multiphoton Magnetooptical Trap, Phys. Rev. Lett. 103, 173003 (2009).

[27] V. Zehnlé and J. C. Garreau, Continuous-Wave Doppler Cooling of Hydrogen Atoms with Two-Photon Transitions, Phys. Rev. A 63, 021402(R) (2001).

[28] D. Sheng, A. Pérez Galván, and L. A. Orozco, Lifetime Measurements of the 5d States of Rubidium, Phys. Rev. A 78, 062506 (2008).

[29] S. Reinhardt, E. Peters, T. W. Hänsch, and T. Udem, Two-Photon Direct Frequency Comb Spectroscopy with Chirped Pulses, Phys. Rev. A 81, 033427 (2010).

[30] P. D. Lett, W. D. Phillips, S. L. Rolston, C. E. Tanner, R. N. Watts, and C. I. Westbrook, Optical Molasses, J. Opt. Soc. Am. B 6, 2084 (1989).

[31] B. C. Duncan, V. Sanchez-Villicana, P. L. Gould, and H. R. Sadeghpour, Measurement of the $\operatorname{Rb}\left(5 D_{5 / 2}\right)$ Photoionization Cross Section Using Trapped Atoms, Phys. Rev. A 63, 043411 (2001).

[32] S. D. Hogan, A. W. Wiederkehr, H. Schmutz, and F. Merkt, Magnetic Trapping of Hydrogen after Multistage Deceleration, Phys. Rev. Lett. 101, 143001 (2008).

[33] M. T. Hummon, W. C. Campbell, H.-I Lu, E. Tsikata, Y. Wang, and J. M. Doyle, Magnetic Trapping of Atomic Nitrogen $\left({ }^{14} N\right)$ and Cotrapping of $\mathrm{NH}\left(X^{3} \Sigma^{-}\right)$, Phys. Rev. A 78, 050702(R) (2008).

[34] T. Kanai, X. Wang, S. Adachi, S. Watanabe, and C. Chen, Watt-Level Tunable Deep Ultraviolet Light Source by a KBBF Prism-Coupled Device, Opt. Express 17, 8696 (2009).

[35] O. Pronin, M. Seidel, F. Lücking, J. Brons, E. Fedulova, M. Trubetskov, V. Pervak, A. Apolonski, T. Udem, and F. Krausz, High-Power Multi-Megahertz Source of WaveformStabilized Few-Cycle Light, Nat. Commun. 6, 6988 (2015). 